\title{
Another role for leptin?
}

Depression and obesity are both serious and expensive health problems worldwide. Now, imbalances in one hormone - leptin - have been implicated in both. Leptin's effect on body weight regulation has already been well documented; recent research from The University of Texas, USA, has shown that it can also attenuate depression-like behaviour in rats.

Lu and colleagues found significant reductions in plasma leptin levels in rats that had undergone chronic unpredictable stress or chronic social defeat. Rats in the former group also showed a concomitant increase in corticosterone, the human equivalent of cortisol, elevations of which are often seen in human patients with depression. When leptin was administered to the chronically stressed rats, it attenuated the hedonic deficit that is associated with chronic stress. However, no effect was seen in non-stressed rats.

The authors then used the forced swim test, which measures 'despair' behaviour - characterized by cessation of escape behaviours such as climbing and swimming - and is commonly used to investigate the efficacy of antidepressant drugs. They found that, in rats subjected to this test, systemic administration of leptin diminished immobility in a dosedependent manner and increased swimming times - in short, it decreased despair behaviour. The positive control, desipramine (a tricyclic antidepressant that selectively inhibits the reuptake of noradrenaline), also reduced rats' immobility in this test, but resulted in increased climbing rather than swimming, indicating that leptin has a different mode of action from the noradrenaline reuptake inhibitors.

Lu and co-workers went on to use in situ hybridization to investigate whether the altered behaviour seen in the forced swim test after leptin administration was accompanied by changes in brain activation. Measuring c-fos mRNA expression, they found increased activity in areas of the hippocampus that included CA1, CA3 and the dentate gyrus, and in the amygdala - most notably in the basolateral nucleus. This suggests that particular limbic structures could be responsible for leptin's effects in this test. Intrahippocampal infusion of leptin during forced swimming produced similar results to systemic administration, indicating that the hippocampus is crucial in meditating leptin's antidepressant-like effects. Meanwhile, intrahypothalamic leptin infusion did not affect behaviour in the forced swim test, indicating that its antidepressant effect is not connected to its role in energy homeostasis.

At present, whether leptin is involved in human depression, and the validity of animal models of depression are both controversial. However, these findings are encouraging, and might pave the way for new antidepressant treatments.

\section{Sarah Archibald}

ORIGINAL RESEARCH PAPER Lu, X.-Y. et al. Leptin: a potential novel antidepressant. Proc. Natl Acad. Sci. USA 103, 1593-1598 (2006) FURTHER READING Berton, O. \& Nestler, E. J. New approaches to antidepressant drug discovery: beyond monoamines. Nature Rev. Neurosci. 7, 137-151 (2006) 